\title{
Red Tunable High-Power Narrow-Spectrum External-Cavity Diode Laser Based on Tapered Amplifier
}

\author{
Mingjun Chi ${ }^{1}$, Ole Bjarlin Jensen"1, Götz Erbert ${ }^{2}$, \\ Bernd Sumpf ${ }^{2}$ and Paul Michael Petersen ${ }^{1}$ \\ ${ }^{1}$ Department of Photonics Engineering, Technical University of Denmark, \\ ${ }^{2}$ Ferdinand-Braun-Institut, Leibniz-Institut für Höchstfrequenztechnik, \\ ${ }^{1}$ Denmark \\ ${ }^{2}$ Germany
}

\section{Introduction}

Diffraction-limited high-power narrow-spectrum red diode lasers are attractive for many applications, such as photodynamic therapy, laser display, and as a pump source to generate UV light by second harmonic generation (SHG). High-power, diffraction-limited diode lasers can be realized by the technology of lasers with a tapered gain-region (Kintzer et al., 1993; Donnelly et al., 1998; Wenzel et al., 2003; Paschke et al., 2005; Sumpf et al., 2009; Fiebig et al., 2010). The tapered laser devices can be used in applications where narrowspectrum is not needed such as photodynamic therapy, but for other applications such as a pump source for UV light generation, the spectral quality of these devices has to be improved.

In order to improve the spectral quality of a tapered laser, different techniques are applied, such as a monolithically integrated master oscillator power amplifier by forming Bragg gratings in the semiconductor material (O'Brien et al., 1993, 1997a), injection locking to an external single-mode laser (Goldberg et al., 1993; Mehuys et al., 1993b; O'Brien et al., 1997b; Wilson et al., 1998; Ferrari et al., 1999; Spießberger et al., 2011), and different external-cavity feedback techniques (Jones et al., 1995; Cornwell \& Thomas, 1997; Morgott et al., 1998; Goyal et al., 1998; Pedersen \& Hansen, 2005; Chi et al., 2005; Lucas-Leclin et al., 2008; Tien et al., 2008; Sakai et al., 2009). Up to $1 \mathrm{~W}$ output power at $668 \mathrm{~nm}$ from a Fabry-Perot tapered diode laser was obtained with a beam quality factor of 1.7, and the spectral width was smaller than $0.2 \mathrm{~nm}$ (Sumpf et al., 2007). Around $670 \mathrm{~nm}$, tunable narrow-linewidth diffraction-limited output was also achieved from an injection-locking tapered diode laser system seeded with a single-mode external-cavity diode laser (Häring et al., 2007); the output power was up to $970 \mathrm{~mW}$. A $670 \mathrm{~nm}$ micro-external-cavity tapered diode laser system was demonstrated with a reflecting volume Bragg grating as a feedback element; in continuous wave (CW) mode, more than $0.5 \mathrm{~W}$ output power was obtained, and in pulse mode, $5 \mathrm{~W}$ peak power was obtained with a beam quality factor of 10 and a spectral width below $150 \mathrm{pm}$ (Tien et al., 2008). Up to $1.2 \mathrm{~W}$ output power at $675 \mathrm{~nm}$ from a tapered laser 
was obtained with a beam quality factor less than 1.3, the maximum conversion efficiency of $31 \%$ was reached at an output power of $1 \mathrm{~W}$ (Sumpf et al., 2011). External-cavity feedback based on a bulk diffraction grating in the Littrow configuration is a useful technique to achieve a tunable narrow-spectrum, high-power, diffraction-limited tapered diode laser system (Mehuys et al., 1993a; Jones et al., 1995; Goyal et al., 1997; Morgott et al., 1998; Chi et al., 2005). We have demonstrated such a tapered diode laser system around $668 \mathrm{~nm}$ with output power up to $810 \mathrm{~mW}$; a beam quality factor of 3.4 was obtained with an output power of $600 \mathrm{~mW}$ (Chi et al., 2009).

In this chapter, three red tunable high-power narrow-spectrum diode laser systems based on three different tapered semiconductor optical amplifiers in Littrow external-cavity are demonstrated. Tapered device A is a $668 \mathrm{~nm}$ 2-mm-long tapered amplifier with a 0.5-mmlong index-guided ridge-waveguide section. Both tapered device $B$ and $C$ are $675 \mathrm{~nm} 2-\mathrm{mm}$ long tapered amplifier, the lengths of ridge-waveguide section are $0.5 \mathrm{~mm}$ for device $\mathrm{B}$, and $0.75 \mathrm{~mm}$ for device $\mathrm{C}$, respectively. The epitaxial structruce and the geometry of these tapered devices are described, and the data on the gain media of the devices are presented and compared.

Laser system A based on device A is tunable over a range of $16 \mathrm{~nm}$ centered at $668 \mathrm{~nm}$. As high as $1.38 \mathrm{~W}$ output power is obtained at $668.35 \mathrm{~nm}$. The emission spectral bandwidth is less than $0.07 \mathrm{~nm}$ throughout the tuning range, and the beam quality factor $M^{2}$ is 2.0 with an output power of $1.27 \mathrm{~W}$.

Laser system B based on device B is tunable from 663 to $684 \mathrm{~nm}$ with output power higher than $0.55 \mathrm{~W}$ in the tuning range, as high as $1.25 \mathrm{~W}$ output power is obtained at $675.34 \mathrm{~nm}$. The emission spectral bandwidth is less than $0.05 \mathrm{~nm}$ throughout the tuning range, and the beam quality factor $M^{2}$ is 2.07 at an output power of $1.0 \mathrm{~W}$. Laser system $C$ based on device $\mathrm{C}$ is tunable from 666 to $685 \mathrm{~nm}$. As high as $1.05 \mathrm{~W}$ output power is obtained around 675.67 $\mathrm{nm}$. The emission spectral bandwidth is less than $0.07 \mathrm{~nm}$ throughout the tuning range, and the beam quality factor $M^{2}$ is 1.13 at an output power of $0.93 \mathrm{~W}$.

The properties of the three tapered diode laser systems are summarized and compared. As an example of application, Laser system $C$ is used as a pump source for the generation of $337.6 \mathrm{~nm}$ UV light by single-pass frequency doubling in a bismuth triborate (BIBO) crystal. An output power of $109 \mu \mathrm{W}$ UV light, corresponding to a conversion efficiency of $0.026 \% \mathrm{~W}^{-1}$ is attained.

\section{Description of the gain media and the tapered devices}

Tapered diode amplifiers (TDAs) consist of an index-guided ridge-waveguide section and a gain-guided tapered section (Kintzer et al., 1993; Sumpf et al., 2009). The ridge-waveguide section works as a spatial filter, thus diffraction-limited laser beam is available from tapered diode amplifiers, and high output power can be obtained due to the amplification from the gain-guided flared section.

The tapered amplifiers used in the experiment were grown using metal organic vapor phase epitaxy. The epitaxial structure of these tapered diode amplifiers were the same. As active layer, a 5-nm-thick compressively strained single $\mathrm{In}_{1-\mathrm{x}} \mathrm{Ga}_{\times} \mathrm{P}$ quantum well was used for all the devices. The gallium content $x$ was selected for an emission wavelength in the range between 670 and $680 \mathrm{~nm}$. The single quantum well was embedded in the $500 \mathrm{~nm}$ thick $\mathrm{Al}_{0.36} \mathrm{Ga}_{0.16} \mathrm{InP} p$ - and $n$-waveguide layers. For the $800 \mathrm{~nm} n$-cladding layer $\mathrm{Al}_{0.52} \mathrm{In}_{0.48} \mathrm{P}$ was 
used, the $1000 \mathrm{~nm}$-cladding layer was made of $\mathrm{Al}_{0.85} \mathrm{Ga}_{0.15} \mathrm{As}$, which allowed carbon doping with concentrations in the range of some $10^{18} \mathrm{~cm}^{-3}$ and a standard AlGaAs process. These epitaxial structures were also used for the manufacturing of tapered lasers as described previously (Sumpf et al., 2007, 2011).

Here we should mention that two factors influence the wavelength, i.e., the spectrum, of a tapered amplifier: the composition of the materials of the quantum well (in the red tapered amplifier, the gallium content $x$ in the $\operatorname{In}_{1-x} G_{x} P$ quantum well) and the strain between quantum well and waveguide. The detailed design on the wavelength of a tapered device is based on the semiconductor physics on quantum well, and this is out of the scope of this book chapter.

\begin{tabular}{lll}
\hline \hline & Gain medium A & Gain medium B \\
\hline$\lambda / \mathrm{nm}$ & 668 & 675 \\
\hline$\Theta_{\text {vert }} /{ }^{\circ}(\mathrm{FWHM})$ & 31 & 30 \\
\hline$\Theta_{\text {vert }} /{ }^{\circ}(95 \%$ power $)$ & 50 & 52 \\
\hline$I_{\mathrm{th}} / \mathrm{mA}$ & 330 & 315 \\
\hline$\eta_{\mathrm{D}}$ & 0.66 & 0.70 \\
\hline$T_{0} / \mathrm{K}$ & 110 & 120 \\
\hline$\alpha_{\mathrm{i}} / \mathrm{cm}^{-1}$ & $(1.8 \pm 0.1)$ & $(1.2 \pm 0.1)$ \\
\hline$\eta_{\mathrm{i}}$ & $(0.90 \pm 0.08)$ & $(0.75 \pm 0.02)$ \\
\hline$\Gamma \cdot \mathrm{g}_{0} / \mathrm{cm}^{-1}$ & $(19.6 \pm 0.4)$ & $(19.8 \pm 0.4)$ \\
\hline \hline
\end{tabular}

Table 1. Summary of the data for the gain media used in this study.

Tapered device A was made of gain medium A, and tapered device B and C were made of gain medium $B$. The gain media data were measured for uncoated broad-area devices (BADs) with a cavity length of $1 \mathrm{~mm}$ and a stripe width of $100 \mu \mathrm{m}$ in pulsed mode. The vertical far field angles $\Theta_{\text {vert }}$ for the devices were about $30^{\circ}$ (FWHM) and between $50^{\circ}$ and $52^{\circ}$ (95\% power content). This relatively small vertical far field angle allows the use of standard optics with a moderate numerical aperture to collimate the output beam. The power-current characteristics and the spectra were measured for these BADs, and the threshold current $I_{\mathrm{th}}$, the differential efficiency $\eta_{\mathrm{D}}$, and the characteristic temperature of the threshold current $T_{0}$ were given in table 1 . The threshold currents of gain medium $\mathrm{A}$ and $\mathrm{B}$ were 330 and $315 \mathrm{~mA}$, respectively. The differential efficiency for gain medium A was slightly smaller compared to gain medium B. The characteristic temperatures of the threshold current were 110 and $120 \mathrm{~K}$ for gain medium A and B, respectively.

Assuming a logarithmic dependence of the gain on the current density, from the lengthdependence measurement of threshold current density $j_{\text {th }}$ and slope efficiency $S$, the gain medium data were obtained and given in Table 1. It showed that for medium A the internal loss $\alpha_{\mathrm{i}}=1.8 \mathrm{~cm}^{-1}$ was larger in comparison to medium B with $\alpha_{\mathrm{i}}=1.2 \mathrm{~cm}^{-1}$. The internal efficiency for medium A was with $\eta_{\mathrm{i}}=0.90$ larger than $\eta_{\mathrm{i}}=0.75$ for medium $\mathrm{B}$. The modal gain coefficient $\Gamma \cdot g_{0}$ remained constant for both gain media within the experimental uncertainty.

Based on these gain media, tapered diode amplifiers were processed with total cavity length of $2 \mathrm{~mm}$. The straight index-guided ridge-waveguide section manufactured by reactive ion etching had a length of $0.5 \mathrm{~mm}$ for tapered device A and B, and $0.75 \mathrm{~mm}$ for tapered device 
C. The width of the ridge-waveguide section was $7.5 \mu \mathrm{m}$ for all the three tapered devices. The tapered gain-guided section was defined by ion implantation, and had a length of 1.5 $\mathrm{mm}$ for device $\mathrm{A}$ and $\mathrm{B}$, and $1.25 \mathrm{~mm}$ for device $\mathrm{C}$. The flared angle was $4^{\circ}$ for all the three devices, and the output apertures for tapered amplifier A, B and C were 110, 112 and $95 \mu \mathrm{m}$, respectively.

The tapered amplifier facets were passivated and antireflection coated to achieve mirror reflectivities of $1 \%$ for the front facet, and $5 \times 10^{-4}$ for the rear facet, respectively. The tapered devices were mounted $p$-side down on copper tungsten $(\mathrm{CuW})$ or chemical vapour deposited (CVD) diamond submounts using AuSn solder. These subassemblies were mounted on standard C-mounts.

\section{External-cavity tapered diode laser systems}

In this section, the tunable external-cavity diode laser systems based on the three tapered amplifiers described in section 2 will be demonstrated.

\subsection{External-cavity tapered diode laser system at $668 \mathrm{~nm}$}

The external-cavity configuration employed for diode laser system A is depicted in Fig. 1 (Chi et al., 2010). An aspherical lens of $3.1 \mathrm{~mm}$ focal length with a numerical aperture (NA) of 0.68 is used to collimate the beam from the back facet in both fast and slow axes. The bulk grating is ruled with 1200 grooves/mm and has a blazed wavelength of $750 \mathrm{~nm}$. The grating is mounted in the Littrow configuration and oriented with the lines in the grating parallel to the active region of the amplifier in order to obtain optimum spectral filtering by the narrow aperture of the tapered device in the fast axis direction. A second aspherical lens of $3.1 \mathrm{~mm}$ focal length with an NA of 0.68 is used to collimate the beam from the output facet in the fast axis. Together with a cylindrical lens of $60 \mathrm{~mm}$ focal length, these two lenses collimate the output beam in the slow axis and compensate the astigmatism simultaneously. All the lenses are antireflection coated for the red wavelength. A beam splitter behind the cylindrical lens is used to reflect part of the output beam of the tapered diode laser system as the diagnostic beam, the spectral width and beam quality factor $M^{2}$ are measured in this beam. The output power of the laser system is measured behind the second aspherical lens.

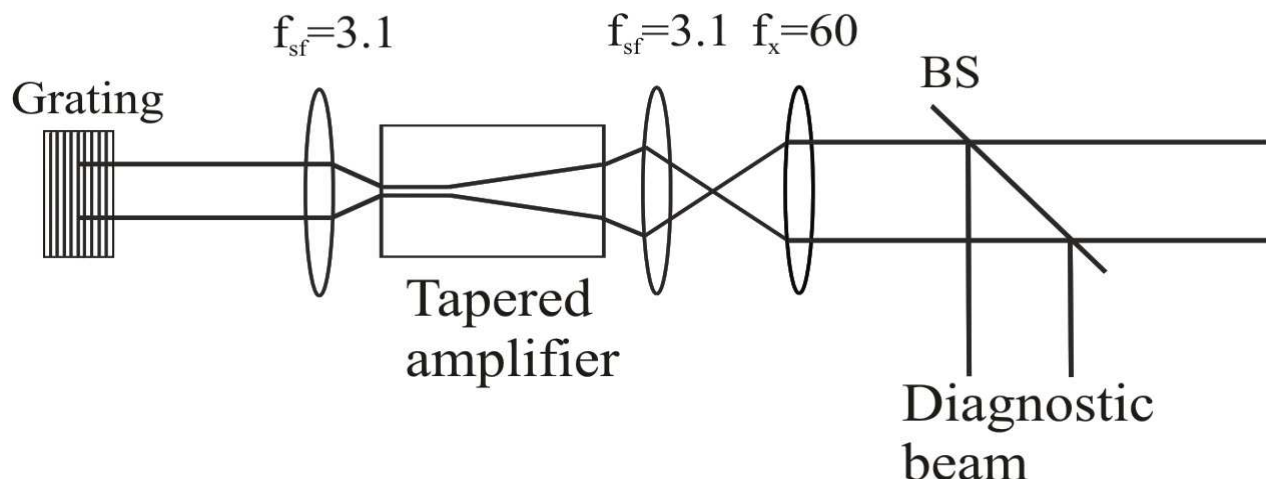

Fig. 1. Experimental setup of tapered diode laser system A using external-cavity feedback. BS, beam splitter. Units are in millimeters. 
The grating is mounted in the Littrow configuration, this means the first-order diffraction beam of the grating propagates back towards the tapered amplifier. Therefore the laser cavity is formed between the diffraction grating and the front facet of the tapered amplifier. The tapered amplifier works as a gain medium in the laser cavity. When the injected current in the tapered device is higher than the threshold, the laser beam oscillates in the laser cavity. The emission wavelength of the laser system is tuned widely by rotating the diffraction grating because of the broad gain bandwidth (a few tens nanometers) of the tapered device. The emission spectrum of the laser system is narrowed significantly compared with the freely running tapered lasers (from a few hundreds picometers to a few tens picometers) due to the dispersion of the diffraction grating and the narrow aperture of the tapered device in the fast axis direction (Sumpf et al., 2007; Chi et al., 2010, 2011).

The laser is TE-polarized, i.e., linearly polarized along the slow axis. The temperature of the amplifier is controlled with a Peltier element and the amplifier is operated at $15{ }^{\circ} \mathrm{C}$ in the experiment. The tapered device lases without the grating feedback.

The power/current characteristics for laser system A with and without the external-cavity feedback are shown in Fig. 2. Without feedback, the threshold current is around $0.7 \mathrm{~A}$, the slope efficiency is $0.63 \mathrm{~W} / \mathrm{A}$, the emission wavelength is around $667.1 \mathrm{~nm}$, the roll-over takes place around $1.5 \mathrm{~A}$, and an output power of $0.65 \mathrm{~W}$ is achieved with an injected current of $2.0 \mathrm{~A}$. With the external-cavity feedback, the maximum power is obtained at a wavelength around $668.4 \mathrm{~nm}$, the threshold current of the laser system is decreased to $0.5 \mathrm{~A}$, the slope efficiency is increased to $1.05 \mathrm{~W} / \mathrm{A}$, the roll-over takes place around $1.7 \mathrm{~A}$, and an output power of $1.38 \mathrm{~W}$ is obtained with an operating current of $2.0 \mathrm{~A}$.

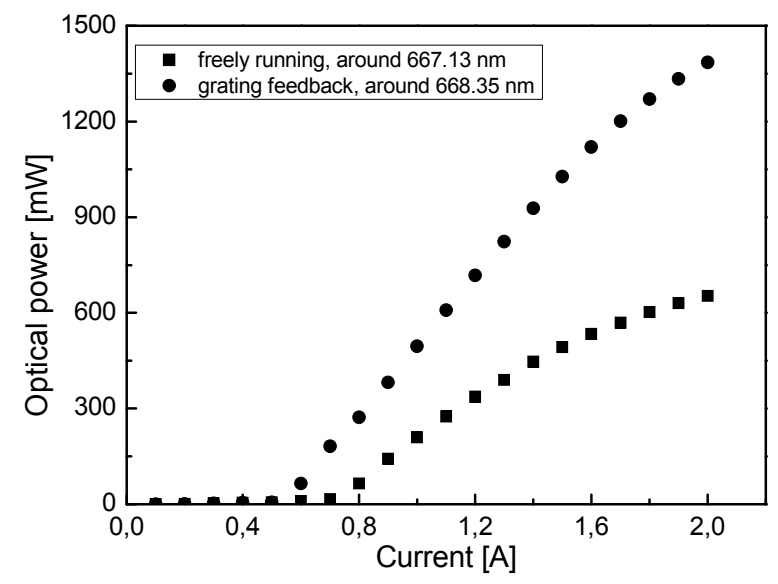

Fig. 2. The power/current characteristics for tapered diode laser system A with and without external-cavity feedback.

The output power at different wavelengths is shown in Fig. 3 at an operating current of 1.8 A. A maximum output power of $1.27 \mathrm{~W}$ is obtained at the wavelength of $668.38 \mathrm{~nm}$, the output power is higher than $0.8 \mathrm{~W}$ in the tuning range from 659 to $675 \mathrm{~nm}$. The tunable range is narrower compared with that in Ref. (Chi et al., 2009), since if we rotate the diffraction grating further to tune the wavelength, the laser system will jump to the freely running mode. 


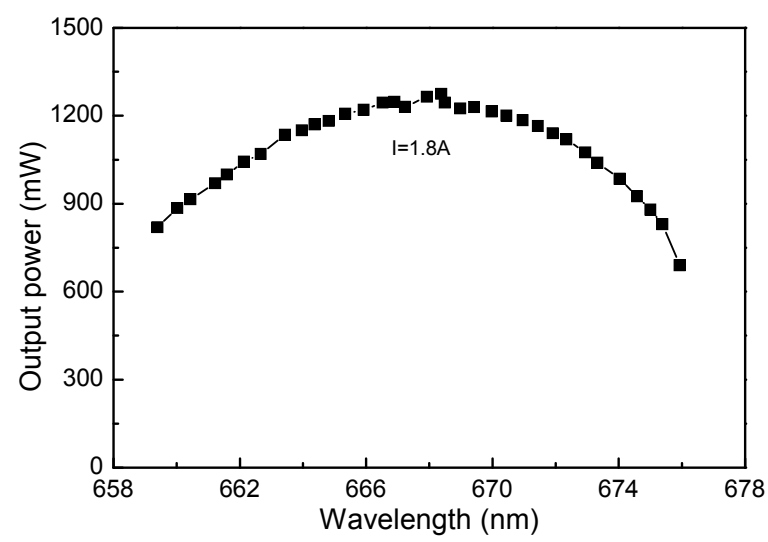

Fig. 3. Tuning curve of tapered diode laser system A at an operating current of $1.8 \mathrm{~A}$.

The spatial beam quality of the output beam along the slow axis is determined by measuring the beam quality factor $M^{2}$ for the tunable external-cavity diode laser system. A spherical lens with a $100 \mathrm{~mm}$ focal length is used to focus the diagnostic beam. Then the beam width, $W\left(1 / \mathrm{e}^{2}\right)$, is measured at various recorded positions $Z$ along the optical axis - on both sides of the beam waist. The value of $M^{2}$ is obtained by fitting the measured data with the formula (Siegman \& Townsend, 1993; Chi et al., 2004):

$$
W=\left[W_{0}^{2}+\left(\frac{4 \lambda M^{2}}{\pi W_{0}}\right)^{2}\left(Z-Z_{0}\right)^{2}\right]^{1 / 2} .
$$

Here $W_{0}$ is the beam waist width, $\lambda$ is the wavelength of the laser system and $Z_{0}$ is the beam waist position.

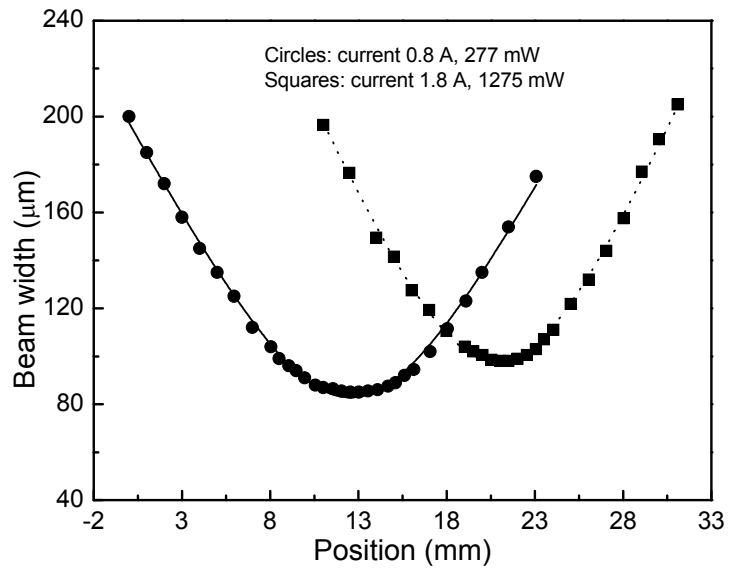

Fig. 4. Beam width of the output beam from tapered diode laser system A for the slow axis with an output power of $277 \mathrm{~mW}$ (circles and solid curve), and $1275 \mathrm{~mW}$ (squares and dotted curve). The curves represent the fits to the measured data using formula (1). 
Figure 4 shows the measured beam widths of the output beam and the fitted curves using formula (1) with the output power of 277 and $1275 \mathrm{~mW}$. The $M^{2}$ values are $1.39 \pm 0.01$ and $2.00 \pm 0.01$ with the output power of 277 and $1275 \mathrm{~mW}$, respectively. For clarity, we have shifted the spatial position of the curves in the figure. In the experiments, the beam waists of the output beam with these two different output powers are located almost at the same position. This means that the astigmatism of the tapered device is rather stable over the whole power range.

The optical spectrum characteristic of the output beam from diode laser system $\mathrm{A}$ is measured using a spectrum analyzer (Advantest Corp. Q8347). A typical result measured at $667.91 \mathrm{~nm}$ with an output power of $1260 \mathrm{~mW}$ is shown in Fig. 5. The figure shows diode laser system A is operated in multiple longitudinal modes. The spectral bandwidth (FWHM) is $0.034 \mathrm{~nm}$ (the resolution of the spectrum analyzer is $3 \mathrm{pm}$ ), and the amplified spontaneous emission intensity is more than $20 \mathrm{~dB}$ suppressed. We find the spectral bandwidth of the output beam is less than $0.07 \mathrm{~nm}$ in the $16 \mathrm{~nm}$ tunable range.

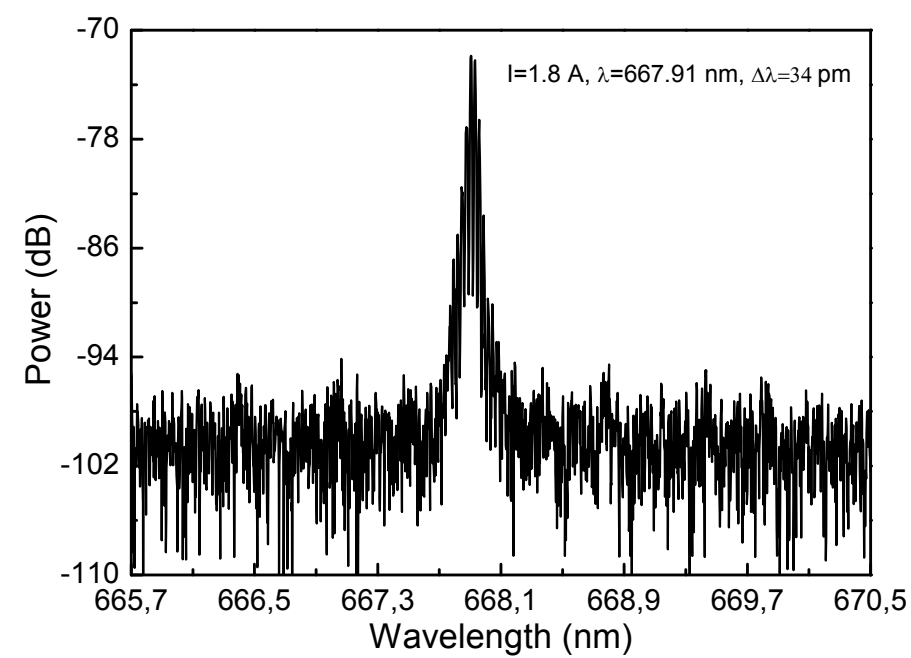

Fig. 5. The optical spectrum of the output beam from tapered diode laser system A with an output power of $1.26 \mathrm{~W}$.

Compared with the results obtained previously in the similar wavelength range (Chi et al., 2009), the output power and spatial beam quality of the tapered diode laser system described above are improved significantly. The reason is the tapered semiconductor amplifier used here has improved properties. The length of the ridge-waveguide section of the $2 \mathrm{~mm}$ tapered device is $0.5 \mathrm{~mm}$ instead of $0.75 \mathrm{~mm}$ in the previous device. It has been verified that the beam quality of the $650 \mathrm{~nm}$ tapered diode lasers with shorter ridgewaveguide section is better compared with the device with longer ridge-waveguide section (Adamiec et al., 2009). This may be also the reason for better beam quality obtained here. The internal loss of the present device is less than $2.0 \mathrm{~cm}^{-1}$, while the internal loss of the previous device is between 2.5 and $3.0 \mathrm{~cm}^{-1}$ (Sumpf et al., 2007). We believe that the lower internal loss is the reason for higher output power from the present external-cavity tapered diode laser system. 


\subsection{External-cavity tapered diode laser systems at $675 \mathrm{~nm}$}

The external-cavity configuration is the same for both tapered gain device B and C as depicted in Fig. 6 (Chi et al., 2011). The detailed description on the external-cavity tapered diode laser system can be found in section 3.1. An aspherical lens is used to collimate the beam from the back facet. A bulk grating is mounted in the Littrow configuration, and the laser cavity is formed between the diffraction grating and the front facet of the tapered amplifier. A second aspherical lens and a cylindrical lens are used to collimate the beam from the output facet. All the lenses are antireflection coated for the red wavelength. A beam splitter behind the cylindrical lens is used to reflect part of the output beam of the tapered diode laser system as the diagnostic beam, where the spectral bandwidth and the beam quality factor $M^{2}$ are measured. The output power of the laser system is measured behind the second aspherical lens.

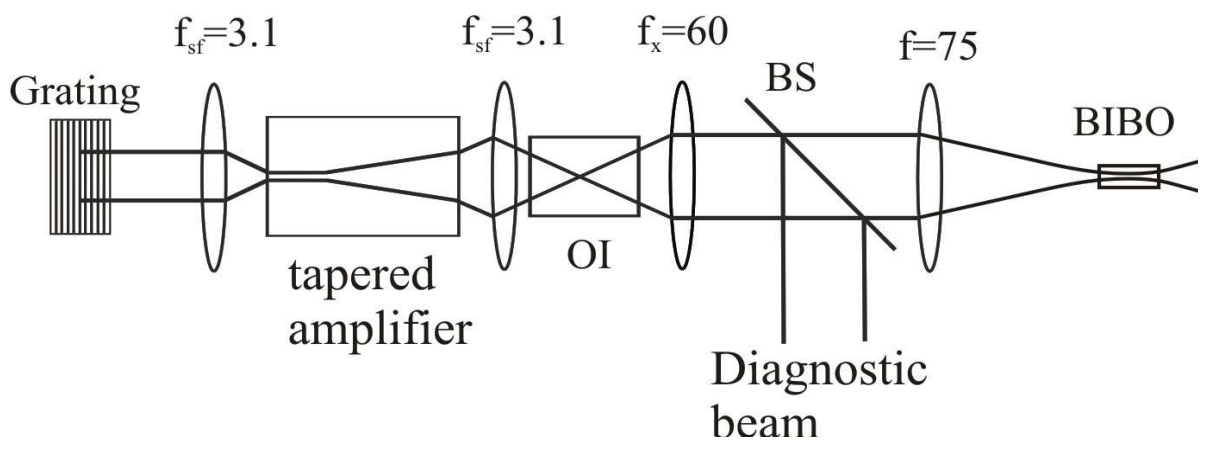

Fig. 6. Experimental setup of the external-cavity tapered diode laser system for SHG. BS, beam splitter; OI, optical isolator. Units are in millimeters.

The lasers are TE-polarized, i.e., linearly polarized along the slow axis. The temperature of the amplifiers is controlled with a Peltier element and the tapered amplifiers are operated at $20{ }^{\circ} \mathrm{C}$ in the experiment. The emission wavelength of the laser systems is tuned by rotating the diffraction grating.

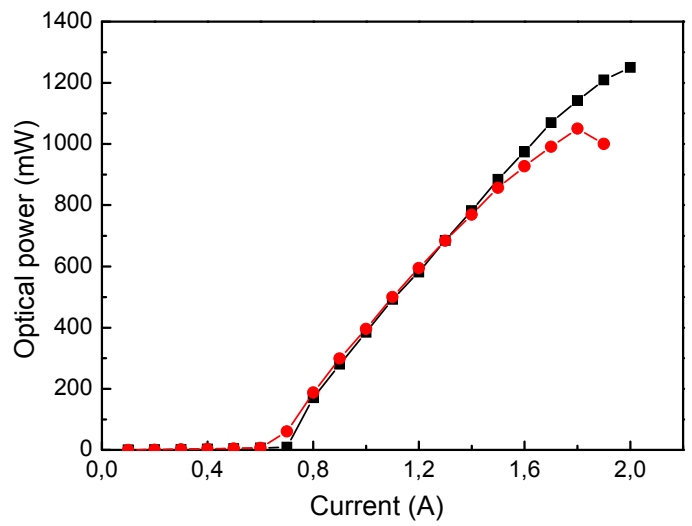

Fig. 7. The power/current characteristics for tapered diode laser system B (squares) and C (circles). 
The power/current characteristics for these two tunable diode laser systems are shown in Fig. 7. For diode laser system $B$, the maximum output power is obtained at the wavelength $675.34 \mathrm{~nm}$, the threshold current is around $0.7 \mathrm{~A}$, the slope efficiency is $1.03 \mathrm{~W} / \mathrm{A}$, the rollover takes place around $1.7 \mathrm{~A}$, and an output power of $1.25 \mathrm{~W}$ is achieved with an injected current of $2.0 \mathrm{~A}$. For diode laser system $\mathrm{C}$, the output power is measured at the wavelength of $675.67 \mathrm{~nm}$, the threshold current of the laser system is around $0.6 \mathrm{~A}$, the slope efficiency is $0.99 \mathrm{~W} / \mathrm{A}$, the roll-over takes place around $1.4 \mathrm{~A}$, an output power of $1.05 \mathrm{~W}$ is obtained with an operating current of $1.8 \mathrm{~A}$, and the output power decreases with higher injected current.

The output power at different wavelengths for these two laser systems is shown in Fig. 8 . For laser system B, at an operating current of $2.0 \mathrm{~A}$, a maximum output power of $1250 \mathrm{~mW}$ is obtained at the wavelength of $675.34 \mathrm{~nm}$. The output power is higher than $550 \mathrm{~mW}$ in the tuning range from 663 to $684 \mathrm{~nm}$. For laser system C, a maximum output power of $1055 \mathrm{~mW}$ is obtained at the wavelength of $675.67 \mathrm{~nm}$; the laser system is tunable from 666 to $685 \mathrm{~nm}$ with output power higher than $460 \mathrm{~mW}$.

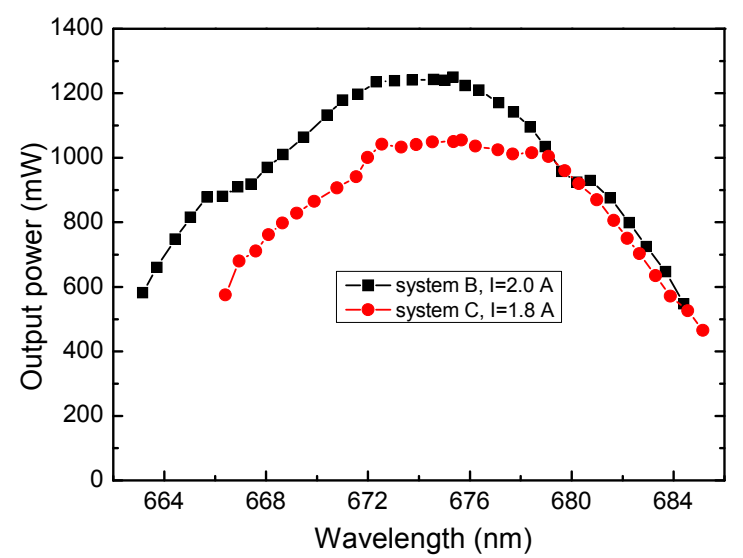

Fig. 8. Tuning curves of the tapered diode laser system B (squares) at an operating current of 2.0 A and system C (circles) at an operating current of $1.8 \mathrm{~A}$.

For both diode laser system B and C, the spatial beam quality of the output beam along the fast axis is assumed to be diffraction-limited because of the waveguide structure of the tapered gain devices. The spatial beam quality of the output beam along the slow axis is determined by measuring the beam quality factor $M^{2}$. A spherical lens with a $65 \mathrm{~mm}$ focal length is used to focus the diagnostic beam. Then the beam width, $W\left(1 / \mathrm{e}^{2}\right)$, is measured at various recorded positions along the optical axis - on both sides of the beam waist. The value of $M^{2}$ is obtained by fitting the measured data with formula (1). Figure 9 shows the measured beam widths and the fitted curves for tapered diode laser system B at output powers of 385 and $1000 \mathrm{~mW}$, where the $M^{2}$ values are $1.28 \pm 0.02$ and $2.07 \pm 0.02$, respectively. Figure 10 shows the measured beam widths and the fitted curves for tapered diode laser system $C$ with the output power of 390 and $930 \mathrm{~mW}$. The $M^{2}$ values are $1.20 \pm$ 0.02 and $1.13 \pm 0.02$ at output powers of 390 and $930 \mathrm{~mW}$, respectively. For clarity, we have shifted the spatial position of the curves in the figures. In the experiments, the waists of the 
output beam with different output powers are located almost at the same position. This indicates that the change of the astigmatism of the tapered device with different injection currents is negligible.

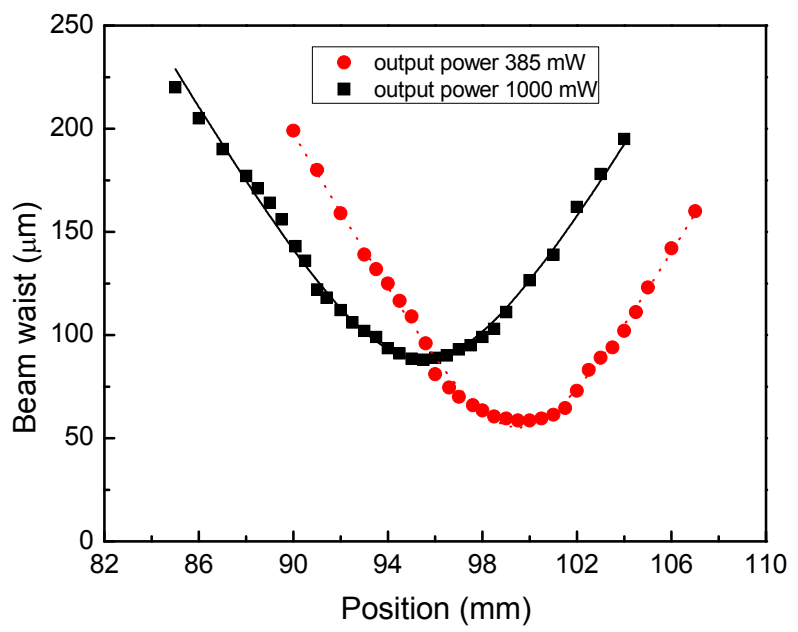

Fig. 9. Beam width of the output beam for the slow axis from diode laser system $B$ with the output power of $385 \mathrm{~mW}$ (circles and dotted curve) and $1000 \mathrm{~mW}$ (squares and solid curve). The curves represent the fits to the measured data using formula (1).

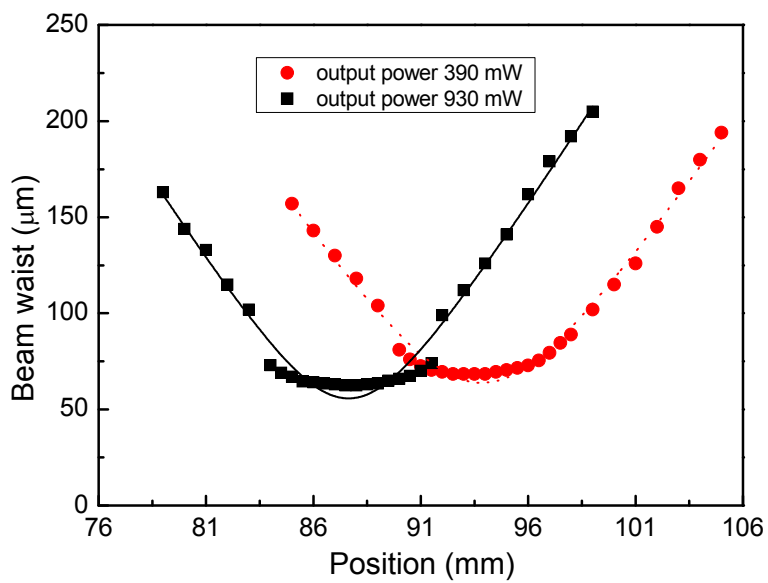

Fig. 10. Beam width of the output beam for the slow axis from diode laser system $C$ with the output power of $390 \mathrm{~mW}$ (circles and dotted curve) and $930 \mathrm{~mW}$ (squares and solid curve). The curves represent the fits to the measured data using formula (1).

The optical spectrum characteristic of the output beam from diode laser system B and C is measured using a spectrum analyzer (Advantest Corp. Q8347). A typical result measured 
for laser system $C$ at $675.04 \mathrm{~nm}$ with an output power of $930 \mathrm{~mW}$ is shown in Fig. 11. The figure shows that the diode laser system is operated in multiple longitudinal modes. The spectral bandwidth (FWHM) is $0.038 \mathrm{~nm}$ (the resolution of the spectrum analyzer is $3 \mathrm{pm}$ ), and the amplified spontaneous emission intensity is more than $20 \mathrm{~dB}$ suppressed. We find the spectral bandwidths of the output beams for diode laser system B and C are less than 0.05 and $0.07 \mathrm{~nm}$ throughout their tunable ranges, respectively.

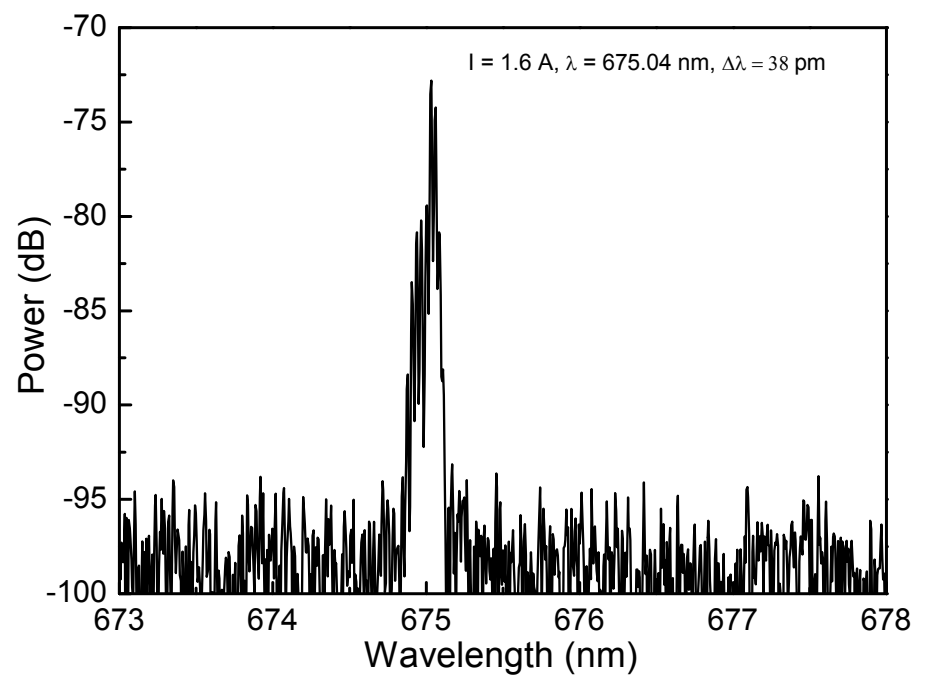

Fig. 11. Optical spectrum of the output beam from tapered diode laser system $\mathrm{C}$ with an output power of $930 \mathrm{~mW}$.

Two tunable high-power $675 \mathrm{~nm}$ external-cavity diode laser systems based on tapered semiconductor optical amplifiers are demonstrated in this subsection. The main parameters of these two diode laser systems are summarized in Table 2; the results of diode laser system A described in subsection 3.1 are also listed in the table for comparison. Diode laser system B can produce more output power than diode laser system $C$, but the spatial beam quality of diode laser system $C$ along the slow axis is significantly better than that of diode laser system B, especially with high output power. The two tapered devices are from the same wafer, so the different behaviour of the two laser systems is originated from the different sizes of the ridge-waveguide section and the tapered section. Diode laser system B produces higher output power since device B has a longer tapered gain section, but this laser system has worse spatial beam quality due to insufficient filtering of the light in the shorter ridgewaveguide section.

Compared with the results from diode laser system $\mathrm{A}$ in subsection 3.1, much more output power around $675.5 \mathrm{~nm}$ is obtained from laser system B and C, i.e., 1.25 and 1.05 $\mathrm{W}$ vs. $0.83 \mathrm{~W}$; and diode laser system A cannot reach wavelengths longer than $676 \mathrm{~nm}$. 
Furthermore, we compare the results from the three laser systems based on different tapered gain devices. This is important for us to choose tapered gain devices for different applications.

\begin{tabular}{lccc}
\hline \hline & \multicolumn{3}{c}{ Laser system } \\
\cline { 2 - 4 } Parameter & $\mathrm{A}$ & $\mathrm{B}$ & $\mathrm{C}$ \\
\hline Max. power $(\mathrm{W})$ & 1.38 & 1.25 & 1.05 \\
\hline $\begin{array}{l}\text { Wavelength with max } \\
\text { power }(\mathrm{nm})\end{array}$ & 668.35 & 675.34 & 675.67 \\
\hline Tunable range $(\mathrm{nm})$ & $659-675$ & $663-684$ & $666-685$ \\
\hline$M^{2}$ value & $2.00 \pm 0.01(1.27 \mathrm{~W})$ & $2.07 \pm 0.02(1.0 \mathrm{~W})$ & $1.13 \pm 0.02(0.93 \mathrm{~W})$ \\
\hline $\begin{array}{l}\text { Spectral bandwidth } \\
(\mathrm{nm})\end{array}$ & $<0.07$ & $<0.05$ & $<0.07$ \\
\hline \hline
\end{tabular}

Table 2. Summary of the main parameters for diode laser system A, B and C.

\section{UV light generation by SHG}

UV light sources are interesting in many fields such as biophotonics/chemical photonics, material processing, and optical data storage. Although diode lasers based on AlGaN have been demonstrated around $340 \mathrm{~nm}$ in pulse mode recently (Yoshida et al., 2008a, 2008b), frequency doubling of a red laser beam through a nonlinear crystal is still an efficient method to generate CW light in this wavelength range (Mizuuchi \& Yamamoto, 1996; Mizuuchi et al., 1997, 2003a, 2003b; Knappe et al., 1998). UV light around $340 \mathrm{~nm}$ has been achieved by single pass SHG in bulk periodically poled $\mathrm{LiTaO}_{3}$ (Mizuuchi \& Yamamoto, 1996; Mizuuchi et al., 1997) and $\mathrm{MgO}: \mathrm{LiNbO}_{3}$ (Mizuuchi et al., 2003a) crystals and also achieved in a periodically poled $\mathrm{MgO}: \mathrm{LiNbO}_{3}$ ridge waveguide (Mizuuchi et al., 2003b); but so far these periodically poled devices are not commercially available, and no UV light shorter than $340 \mathrm{~nm}$ has been demonstrated with these first-order periodically poled devices.

Here UV light around $337.5 \mathrm{~nm}$ will be generated using the external-cavity tapered diode laser system developed above as a pump source. The generated CW UV light source will be used as the excitation source for fluorescence diagnostics. Compared with other UV laser sources around $337 \mathrm{~nm}$, such as a CW krypton-ion laser and a pulsed nitrogen laser, the UV laser source based on a tunable tapered diode laser system is far more simple, compact, and easy to operate.

A BIBO nonlinear crystal is used for frequency doubling of the $675.2 \mathrm{~nm}$ red light to the $337.6 \mathrm{~nm}$ UV light due to its relatively high effective nonlinear coefficient. The $10 \mathrm{~mm}$ long BIBO crystal with an aperture of $4 \mathrm{~mm} \times 4 \mathrm{~mm}$ is cut with $\theta=137.7^{\circ}$ and $\varphi=90^{\circ}$ for type-I phase matching (eeo) and antireflection coated on both end surfaces for $675 / 337.5 \mathrm{~nm}$. The spectral bandwidth of both laser system $B$ and $C$ is narrow enough for frequency doubling through the BIBO crystal (the acceptable spectral bandwidth of this crystal is around 0.2 $\mathrm{nm}$ ). Laser system $\mathrm{C}$ is chosen as the pump source for the frequency doubling experiment due to its better spatial beam quality. 
A $30 \mathrm{~dB}$ optical isolator is inserted between the aspherical lens and the cylindrical lens in the output beam to avoid feedback from the optical components and the nonlinear crystal, as shown in Fig. 6. A biconvex lens of $75 \mathrm{~mm}$ focal length is used to focus the red fundamental beam into the BIBO crystal. The available output power of the fundamental beam in front of the crystal is $650 \mathrm{~mW}$. The size of the focus $w_{\mathrm{s}} \times w_{\mathrm{f}}$ is around $70 \mu \mathrm{m} \times 35 \mu \mathrm{m}$, where $w_{\mathrm{s}}$ and $w_{\mathrm{f}}$ are the beam waists (diameters at $1 / \mathrm{e}^{2}$ ) in the slow and fast axes, respectively. The elliptical beam is used to reduce the effects of walk-off in the BIBO crystal. The walk-off angle in our crystal is $72.9 \mathrm{mrad}$, corresponding to a heavy walk-off parameter $B$ of 15.1 . The elliptical beam was proved to be optimum in the experiments, in good agreement with the theory of frequency doubling using elliptical beams (Boyd \& Kleinman, 1968; Steinbach et al., 1996). The slight change in astigmatism with output power will cause the focusing conditions to vary slightly at different power levels. In the experiments, the astigmatism was corrected at maximum pump power. Two dichroic beam splitters separate the fundamental beam from the second harmonic output beam.

The wavelength of the fundamental beam is tuned to $675.16 \mathrm{~nm}$, and the temperature of the crystal is $19.8{ }^{\circ} \mathrm{C}$. Figure 12 shows the measured second harmonic power as a function of fundamental power. The curve represents a quadratic fitting. A maximum of $109 \mu \mathrm{W} \mathrm{UV}$ light is obtained with a fundamental pump power of $650 \mathrm{~mW}$. The conversion efficiency $\eta$ is $0.026 \% \mathrm{~W}^{-1}$, compared to a conversion efficiency of $0.019 \% \mathrm{~W}^{-1}$ for a single-pass frequency doubling through a $15 \mathrm{~mm}$ long $\mathrm{LiIO}_{3}$ bulk crystal (Knappe et al., 1998), and the theoretically calculated value is $0.040 \% \mathrm{~W}^{-1}$ (Steinbach et al., 1996).

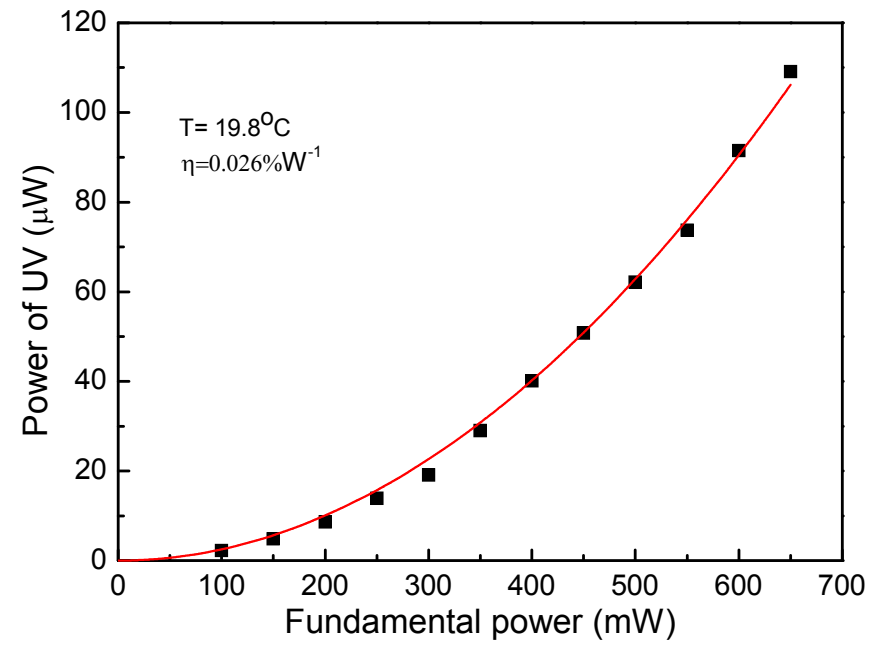

Fig. 12. Second harmonic power as a function of fundamental power. The squares are measured data, and the curve is a quadratic fit. 


\section{Conclusion}

In conclusion, three tunable high-power narrow-spectrum red diode laser systems based on tapered semiconductor optical amplifiers in Littrow external-cavity are demonstrated. The epitaxial structruce and the geometry of these tapered devices are described, and the data on the gain media of the devices are presented and compared.

For diode laser system A at $668 \mathrm{~nm}$, an output power of $1.38 \mathrm{~W}$ is obtained with an injected current of $2.0 \mathrm{~A}$, and the laser system is tunable from 659 to $675 \mathrm{~nm}$ with output power over $800 \mathrm{~mW}$. This is to our knowledge the highest output power from a tunable diode laser system in this wavelength range. The spectral bandwidth of the output beam is less than $0.07 \mathrm{~nm}$.

Both diode laser system $B$ and $C$ are tunable in a $20 \mathrm{~nm}$ range centered at $675 \mathrm{~nm}$, and the spectral bandwidth of the output beam for both diode laser systems is less than $0.07 \mathrm{~nm}$ in their tunable ranges. The maximum output power is $1.25 \mathrm{~W}$ obtained from laser system $\mathrm{B}$ at the wavelength of $675.3 \mathrm{~nm}$, and the maximum output power from laser system $\mathrm{C}$ is $1.05 \mathrm{~W}$ obtained at the wavelength of $675.6 \mathrm{~nm}$. The beam quality factor $M^{2}$ is 2.07 with the output power of $1.0 \mathrm{~W}$ for laser system $\mathrm{B}$, and the $M^{2}$ value is 1.13 with the output power of $0.93 \mathrm{~W}$ for laser system $C$. Laser system $C$ is used as the pump source for the generation of UV light by single-pass frequency doubling in a BIBO crystal. An output power of $109 \mu \mathrm{W}$ UV light at $337.6 \mathrm{~nm}$, corresponding to a conversion efficiency of $0.026 \% \mathrm{~W}^{-1}$ is attained.

\section{Acknowledgment}

The authors acknowledge the financial support of the European community through the project WWW.BRIGHTER.EU (grant No. FP6-IST-035266).

\section{References}

Adamiec, P.; Sumpf, B.; Rüdiger, I.; Fricke, J.; Hasler, K.-H.; Ressel, P.; Wenzel, H.; Zorn, M.; Erbert, G. \& Tränkle, G. (2009). Tapered lasers emitting at $650 \mathrm{~nm}$ with $1 \mathrm{~W}$ output power with nearly diffraction-limited beam quality. Opt. Lett., Vol. 34, No. 16, (August 2009), pp. 2456-2458, ISSN 0146-9592

Boyd, G.D. \& Kleinman, D.A. (1968). Parametric interaction of focused Gaussian light beams. J. Appl. Phys., Vol. 39, No. 8, (July 1968), pp. 3597-3639, ISSN 0021-8979

Chi, M.; Bøgh, N.-S.; Thestrup, B. \& Petersen, P.M. (2004). Improvement of the beam quality of a broad-area diode laser using double feedback from two external mirrors. Appl. Phys. Lett., Vol. 85, No. 7, (August 2004), pp. 1107-1109, ISSN 0003-6951

Chi, M.; Jensen, O.B.; Holm, J.; Pedersen, C.; Andersen, P.E.; Erbert, G.; Sumpf, B. \& Petersen, P.M. (2005). Tunable high-power narrow-linewidth semiconductor laser based on an external-cavity tapered amplifier. Opt. Express, Vol. 13, No. 26, (December 2005), pp. 10589-10596, ISSN 1094-4087

Chi, M.; Jensen, O.B.; Erbert, G.; Sumpf, B. \& Petersen, P.M. (2009). Tunable high-power narrow-linewidth semiconductor laser based on an external-cavity tapered amplifier at $670 \mathrm{~nm}$. Proceeding of the $8^{\text {th }}$ Pacific Rim conference on Lasers and 
Electro-optics, WD1-4, ISBN 978-1-4244-3830-3, Shanghai, China, Aug. 30-Sep. 3, 2009

Chi, M.; Erbert, G.; Sumpf, B. \& Petersen, P.M. (2010). Tunable high-power narrow-spectrum external-cavity diode laser based on tapered amplifier at $668 \mathrm{~nm}$. Opt. Lett., Vol. 35, No. 10, (May 2010), pp. 1545-1547, ISSN 0146-9592

Chi, M.; Jensen, O.B.; Erbert, G.; Sumpf, B. \& Petersen, P.M. (2011). Tunable high-power narrow-spectrum external-cavity diode laser at $675 \mathrm{~nm}$ as a pump source for UV generation. Appl. Opt., Vol. 50, No. 1, (January 2011), pp. 90-94, ISSN 0003-6935

Cornwell, D.M. \& Thomas, H.J. (1997). High-power (>0.9 W cw) diffraction-limited semiconductor laser based on a fiber Bragg grating external cavity. Appl. Phys. Lett., Vol. 70, No. 6, (February 1997), pp. 694-695, ISSN 0003-6951

Donnelly, J.P.; Walpole, J.N.; Groves, S.H.; Bailey, R.J.; Missaggia, L.J.; Napoleone, A.; Reeder, R.E. \& Cook, C.C. (1998). 1.5- $\mu \mathrm{m}$ tapered-gain-region lasers with high-CW output powers. IEEE Photon. Technol. Lett., Vol. 10, No. 10, (October 1998), pp. 13771379, ISSN 1041-1135

Ferrari, G.; Mewes, M.; Schreck, F. \& Salomon, C. (1999). High-power multiple-frequency narrow-linewidth laser source based on a semiconductor tapered amplifier. Opt. Lett., Vol. 24, No. 3, (February 1999), pp. 151-153, ISSN 0146-9592

Fiebig, C.; Eppich, B.; Paschke, K. \& Erbert, G. (2010). High-brightness 980-nm tapered laseroptimization of the laser rear facet. IEEE Photon. Technol. Lett., Vol. 22, No. 5, (March 2010), pp. 341-343, ISSN 1041-1135

Goldberg, L.; Mehuys, D.; Surette, M.R. \& Hall, D.C. (1993). High-power, near-diffractionlimited large-area traveling-wave semiconductor amplifiers. IEEE J. Quantum Electron., Vol. 29, No. 6, (June 1993), pp. 2028-2043, ISSN 0018-9197

Goyal, A.K.; Gavrilovic, P. \& Po, H. (1997). Stable single-frequency operation of a highpower external cavity tapered diode laser at $780 \mathrm{~nm}$. Appl. Phys. Lett., Vol. 71, No. 10, (September 1997), pp. 1296-1298, ISSN 0003-6951

Goyal, A.K.; Gavrilovic, P. \& Po, H. (1998). 1.35 W of stable single-frequency emission from an external-cavity tapered oscillator utilizing fiber Bragg grating feedback. Appl. Phys. Lett., Vol. 73, No. 5, (August 1998), pp. 575-577, ISSN 0003-6951

Häring, R.; Sumpf, B.; Erbert, G.; Tränkle, G.; Lison, F. \& Kaenders, W.G. (2007). 670 nm semiconductor lasers for Lithium spectroscopy with $1 \mathrm{~W}$. Proceedings of SPIE, Vol. 6485, 648516, ISBN 978-0-8194-6598-6, San Jose, CA, USA, Jan. 20-25, 2007

Jones, R.J.; Gupta, S.; Jain, R.K. \& Walpole, J.N. (1995). Near-diffraction-limited high power $(\sim 1 \mathrm{~W})$ single longitudinal mode CW diode laser tunable from 960 to 980 nm. Electron. Lett., Vol. 31, No. 19, (September 1995), pp. 1668-1669, ISSN 00135194

Kintzer, E.S.; Walpole, J.N.; Chinn, S.R.; Wang, C.A. \& Missaggia, L.J. (1993). High-power, strained-layer amplifiers and lasers with tapered gain regions. IEEE Photon. Technol. Lett., Vol. 5, No. 6, (June 1993), pp. 605-608, ISSN 1041-1135

Knappe, R.; Laue, C.K. \& Wallenstein, R. (1998). Tunable UV-source based on frequencydoubled red diode laser oscillator-amplifier system. Electron. Lett., Vol. 34, No. 12, (June 1998), pp. 1233-1234, ISSN 0013-5194 
Lucas-Leclin, G.; Paboeuf, D.; Georges, P.; Holm, J.; Andersen, P.; Sumpf, B. \& Erbert, G. (2008). Wavelength stabilization of extended-cavity tapered lasers with volume Bragg gratings. Appl. Phys. B, Vol. 91, No. 3-4, (June 2008), pp. 493-498, ISSN 14320649

Mehuys, D.; Welch, D. \& Scifres, D. (1993a). 1 W CW, diffraction-limited, tunable externalcavity semiconductor laser. Electron. Lett., Vol. 29, No. 14, (July 1993), pp. 12541255, ISSN 0013-5194

Mehuys, D.; Goldberg, L. \& Welch, D.F. (1993b). 5.25-W CW near-diffraction-limited tapered -stripe semiconductor optical amplifier. IEEE Photon. Technol. Lett., Vol. 5, No. 10, (October 1993), pp. 1179-1182, ISSN 1041-1135

Mizuuchi, K. \& Yamamoto, K. (1996). Generation of 340-nm light by frequency doubling of a laser diode in bulk periodically poled $\mathrm{LiTaO}_{3}$. Opt. Lett., Vol. 21, No. 2, (January 1996), pp. 107-109, ISSN 0146-9592

Mizuuchi, K.; Yamamoto, K. \& Kato, M. (1997). Generation of ultraviolet light by frequency doubling of a red laser diode in a first-order periodically poled bulk $\mathrm{LiTaO}_{3}$. Appl. Phys. Lett., Vol. 70, No. 10, (March 1997), pp. 1201-1203, ISSN 0003-6951

Mizuuchi, K.; Morikawa, A.; Sugita, T. \& Yamamoto, K. (2003a). Efficient secondharmonic generation of $340-\mathrm{nm}$ light in a $1.4-\mu \mathrm{m}$ periodically poled bulk MgO:LiNbO3. Jpn. J. Appl. Phys., Vol. 42, No. 2A, (February 2003), pp. L90-L91, ISSN 0021-4922

Mizuuchi, K.; Sugita, T.; Yamamoto, K.; Kawaguchi, T.; Yoshino, T. \& Imaeda, M. (2003b). Efficient 340-nm light generation by a ridge-type waveguide in a first-order periodically poled $\mathrm{MgO}: \mathrm{LiNbO}_{3}$. Opt. Lett., Vol. 28, No. 15, (August 2003), pp. 13441346, ISSN 0146-9592

Morgott, S.; Chazan, P.; Mikulla, M.; Walther, M.; Kiefer, R.; Braunstein, J. \& Weimann, G. (1998). High-power near-diffraction-limited external cavity laser, tunable from 1030 to 1085 nm. Electron. Lett., vol. 34, No. 6, (March 1998), pp. 558-559, ISSN 0013-5194

O’Brien, S.; Welch, D.F.; Parke, R.A.; Mehuys, D.; Dzurko, K.; Lang, R.J.; Waarts, R. \& Scifres, D. (1993). Operating characteristics of a high-power monolithically integrated flared amplifier master oscillator power amplifier. IEEE J. Quantum Electron., Vol. 29, No. 6, (June 1993), pp. 2052-2057, ISSN 0018-9197

O’Brien, S.; Lang, R.; Parke, R.; Major, J.; Welch, D.F. \& Mehuys, D. (1997a). 2.2-W continuous-wave diffraction-limited monolithically integrated master oscillator power amplifier at $854 \mathrm{~nm}$. IEEE Photon. Technol. Lett., Vol. 9, No. 4, (April 1997), pp. 440-442, ISSN 1041-1135

O'Brien, S.; Schoenfelder, A. \& Lang, R.J. (1997b). 5-W CW diffraction-limited InGaAs broad-area flared amplifier at $970 \mathrm{~nm}$. IEEE Photon. Technol. Lett., Vol. 9, No. 9, (September 1997), pp. 1217-1219, ISSN 1041-1135

Paschke, K.; Sumpf, B.; Dittmar, F.; Erbert, G.; Staske, R.; Wenzel, H. \& Tränkle, G. (2005). Nearly diffraction limited 980-nm tapered diode lasers with an output power of 7.7 W. IEEE J. Sel. Top. Quantum Electron., Vol. 11, No. 5, (September/October 2005), pp. 1223-1227, ISSN 1077-260X 
Pedersen, C. \& Hansen, R.S. (2005). Single frequency, high power, tapered diode laser using phase-conjugated feedback. Opt. Express, Vol. 13, No. 11, (May 2005), pp. 3961-3968, ISSN 1094-4087

Sakai, K.; Itakura, S.; Shimada, N.; Shibata, K.; Hanamaki, Y.; Yagi, T. \& Hirano, Y. (2009). High-power tapered unstable-resonator laser diode with a fiber-Bragg grating reflector. IEEE Photon. Technol. Lett., Vol. 21, No. 16, (August 2009), pp. 1103-1105, ISSN 1041-1135

Siegman, A.E. \& Townsend, S.W. (1993). Output beam propagation and beam quality from a multimode stable-cavity laser. IEEE J. Quantum Electron., Vol. 29, No. 4, (April 1993), pp. 1212-1217, ISSN 0018-9197

Spießberger, S.; Schiemangk, M.; Sahm, A.; Wicht, A.; Wenzel, H.; Peters, A.; Erbert, G. \& Tränkle, G. (2011). Micro-integrated 1 Watt semiconductor laser system with a linewidth of $3.6 \mathrm{kHz}$. Opt. Express, Vol. 19, No. 8, (April 2011), pp. 7077-7083, ISSN 1094-4087

Steinbach, A.; Rauner, M.; Cruz, F.C. \& Bergquist, J.C. (1996). CW second harmonic generation with elliptical Gaussian beams. Opt. Commun., Vol. 123, No. 1-3, (January 1996), pp. 207-214, ISSN 0030-4018

Sumpf, B.; Erbert, G.; Fricke, J.; Froese, P.; Häring, R.; Kaenders, W.G.; Klehr, A.; Lison, F.; Ressel, P.; Wenzel, H.; Weyers, M.; Zorn, M. \& Tränkle, G. (2007). 670 nm tapered lasers and amplifiers with output powers $\mathrm{P} \geq 1 \mathrm{~W}$ and nearly diffraction limited beam quality. Proceedings of SPIE, Vol. 6485, 648517, ISBN 978-0-8194-6598-6, San Jose, CA, USA, Jan. 20-25, 2007

Sumpf, B.; Hasler, K.-H.; Adamiec, P.; Bugge, F.; Dittmar, F.; Fricke, J.; Wenzel, H.; Zorn, M.; Erbert, G. \& Tränkle, G. (2009). High-Brightness quantum well tapered lasers. IEEE J. Sel. Top. Quantum Electron., Vol. 15, No. 3, (May/June 2009), pp. 1009-1020, ISSN 1077-260X

Sumpf, B.; Adamiec, P.; Zorn, M.; Wenzel, H. \& Erbert, G. (2011). Nearly Diffraction-limited tapered lasers at $675 \mathrm{~nm}$ with 1-W output power and conversion efficiencies above 30\%. IEEE Photon. Technol. Lett., Vol. 23, No. 4, (February 2011), pp. 266-268, ISSN 1041-1135

Tien, T.Q.; Maiwald, M.; Sumpf, B.; Erbert, G. \& Tränkle, G. (2008). Microexternal cavity tapered lasers at $670 \mathrm{~nm}$ with $5 \mathrm{~W}$ peak power and nearly diffraction-limited beam quality. Opt. Lett., Vol. 33, No. 22, (November 2008), pp. 2692-2694, ISSN 0146-9592

Wenzel, H.; Sumpf, B. \& Erbert, G. (2003). High-brightness diode lasers. Comptes Rendus Physique, Vol. 4, No. 6, (July-August 2003), pp. 649-661, ISSN 1631-0705

Wilson, A.C.; Sharpe, J.C.; McKenzie, C.R.; Manson, P.J. \& Warrington, D.M. (1998). Narrow-linewidth master-oscillator power amplifier based on a semiconductor tapered amplifer. Appl. Opt., Vol. 37, No. 21, (July 1998), pp. 4871-4875, ISSN 00036935

Yoshida, H.; Yamashita, Y.; Kuwabara, M. \& Kan, H. (2008a). A 342-nm ultraviolet AlGaN multiple-quantum-well laser diode. Nat. Photon., Vol. 2, No. 9, (September 2008), pp. 551-554, ISSN 1749-4885 
Yoshida, H.; Yamashita, Y.; Kuwabara, M. \& Kan, H. (2008b). Demonstration of an ultraviolet $336 \mathrm{~nm}$ AlGaN multiple-quantum-well laser diode. Appl. Phys. Lett., Vol. 93, No. 24, (December, 2008), 241106, ISSN 0003-6951 


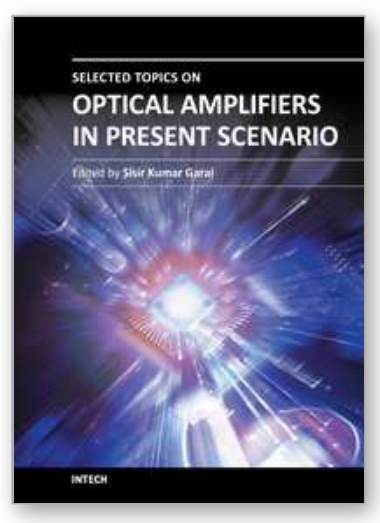

\author{
Selected Topics on Optical Amplifiers in Present Scenario \\ Edited by Dr. Sisir Garai
}

ISBN 978-953-51-0391-2

Hard cover, 176 pages

Publisher InTech

Published online 23, March, 2012

Published in print edition March, 2012

With the explosion of information traffic, the role of optics becomes very significant to fulfill the demand of super fast computing and data processing and the role of optical amplifier is indispensable in optical communication field. This book covers different advance functionalities of optical amplifiers and their emerging applications such as the role of SOA in the next generation of optical access network, high speed switches, frequency encoded all-optical logic processors, optical packet switching architectures, microwave photonic system, etc. Technology of improving the gain and noise figure of EDFA and, the study of the variation of material gain of QD structure are also included. All the selected topics are very interesting, well organized and hope it will be of great value to the postgraduate students, academics and anyone seeking to understand the trends of optical amplifiers in present scenario.

\title{
How to reference
}

In order to correctly reference this scholarly work, feel free to copy and paste the following:

Mingjun Chi, Ole Bjarlin Jensen, Götz Erbert, Bernd Sumpf and Paul Michael Petersen (2012). Red Tunable High-Power Narrow-Spectrum External-Cavity Diode Laser Based on Tapered Amplifier, Selected Topics on Optical Amplifiers in Present Scenario, Dr. Sisir Garai (Ed.), ISBN: 978-953-51-0391-2, InTech, Available from: http://www.intechopen.com/books/selected-topics-on-optical-amplifiers-in-present-scenario/red-tunable-highpower-narrow-spectrum-external-cavity-diode-laser-based-on-tapered-amplifier-

\section{INTECH}

open science | open minds

\section{InTech Europe}

University Campus STeP Ri

Slavka Krautzeka 83/A

51000 Rijeka, Croatia

Phone: +385 (51) 770447

Fax: +385 (51) 686166

www.intechopen.com

\section{InTech China}

Unit 405, Office Block, Hotel Equatorial Shanghai

No.65, Yan An Road (West), Shanghai, 200040, China

中国上海市延安西路65号上海国际贵都大饭店办公楼 405 单元

Phone: +86-21-62489820

Fax: $+86-21-62489821$ 
(C) 2012 The Author(s). Licensee IntechOpen. This is an open access article distributed under the terms of the Creative Commons Attribution 3.0 License, which permits unrestricted use, distribution, and reproduction in any medium, provided the original work is properly cited. 